\title{
Beats produced between a rhythmic applied force and the resting tremor of Parkinsonism
}

\author{
E. G. W A L S H \\ From the Department of Physiology, University of Edinburgh, Edinburgh
}

SUMMARY Rhythmic forces have been applied to the wrist of patients with Parkinsonism tremor by means of a printed motor. The tremor rate was not altered to that of the applied force. On the contrary, beats were established, the rate of which depended on the difference in rate between the tremor and the applied rhythm. Most of the observations have been for horizontal motion of the hand but similar phenomena have been seen for vertical movements, and for other parts of the body-for example, foot, elbow, finger joint, and head. The observations are regarded as supporting the view that the tremorgenic mechanism is central. There was no electromyographic evidence of servo driving or servo assistance in the genesis of the tremor.

The tremor of Parkinsonism is sometimes said to result from self re-excitation of reflexes in the limb (Hufschmidt, 1959, 1963; Stiles and Pozos, 1976). If this were indeed the mechanism it would be expected that the tremor could be entrained. Thus, for a tremor at $5 \mathrm{~Hz}$ the application of an applied force at $4 \mathrm{~Hz}$ or $6 \mathrm{~Hz}$ might be expected to change the rate of the tremor to $4 \mathrm{~Hz}$ or $6 \mathrm{~Hz}$ respectively; furthermore, for small frequency differences quite low levels of applied force should be effective.

The present paper is concerned with this possibility. In an extended series of patients entrainment has not been seen. On the contrary, beats can very often be set up between the natural and the applied rhythm. Preliminary accounts of this work have been published elsewhere (Walsh. 1969, 1978).

\section{Methods}

For the investigation of hand movements, a G9M4 printed motor (Printed Motors Ltd) has been used aligned with the wrist joint. The spindle is double-ended, and the hand has been coupled to one end by a light alloy crank. The fingers are bandaged to the crank with Velcro, and the other end of the motor is coupled to a precision plastic potentiometer which provides a signal of displacement and, by electrical differentiation, of velocity. Most observations have been for horizontal move-

Address for reprint requests: Dr E. G. Walsh. Department of Physiology, Teviot Place, Edinburgh EH8 9AG.

Accepted 25 July 1978 ment but for some vertical motion has been employed, the patient being instructed to keep his hand approximately horizontal. The apparatus has been illustrated elsewhere (Gillingham et al., 1972 Fig. 4, p. 102). With the crank removed and a screwdriver handle taking its place some observations have been made on pronation/supination movements of the forearm.

The same motor working through a lever and linkage has been used to investigate finger tremor and jaw tremor. For the latter the force has been transmitted to a clamp applied between the lower incisor teeth and the underside of the chin. A layer of balsa wood was placed between the clamp and the teeth. For head movements the linkage has been attached to a tightly fitting alloy helmet. Elbow tremor has been investigated using the larger G12M4 printed motor fitted with a suitable lever. The motion was in the horizontal plane.

For ankle movements a G16M4 motor has been used. The method has been described and illustrated elsewhere in this journal (Walsh, 1974, 1976).

In the observations the power amplifier driving the motor has supplied a waveform usually of a sinusoidal shape. Electromyography was performed using suction cup electrodes; in occasional observations fine silver wires have been inserted into the muscles.

An 8 channel Mingograf spray recorder or an SE Laboratories pen writer have been used to record the applied torque, position, velocity, and electromyographic data. In some records the phase between the velocity trace and the applied torque 
have been computed electronically and displayed on one of the channels.

The patients came to the laboratory for assessment of their neurophysiological status before therapy which was either L-dopa or stereotaxic surgery. Included in the series was a substantial number with some degree of rigidity but no definite tremor at rest. These have been excluded from the present report which deals only with patients who had resting tremor, sustained sufficiently well for the investigations to be undertaken. When the tremor was present unilaterally, or was clearly more conspicuous on one side, that side was tested. When, however, the tremor was bilaterally symmetrical the right hand was tested.

\section{Results}

\section{GENERAL OBSERVATIONS}

In many patients the tremor of wrist movement is remarkably sinusoidal. This is true even when the velocity trace is examined-it shows irregularities more clearly than the position recording. The waveform departs from the sinusoidal when the motion is small. The tremor commonly shows considerable but slow variations of amplitude but only small variations of frequency as estimated by counting zero crossings. The tremor may be present only periodically. At times electromyographic activity at the tremor frequency can be clearly detected when there is no overt movement of the joint. On such occasions it may be possible to see a flickering movement of the skin over a part of the muscle which is evidently not nearly powerful enough to move the hand.

The rate of the tremor does not normally vary with amplitude. Thus, when solely detectable by electrical recordings, the rate is the same as when fully developed with the wrist moving substantially. These findings are not in agreement with those of Stiles and Pozos (1976) who claimed an inverse relationship between frequency and amplitude.

It may at times be desirable to bring out the tremor when it is temporarily absent. For this it is useful for the patient to tap a Morse key as rapidly as possible with the opposite hand (Dinakar and Walsh, 1973). Alternatively a foot switch may be provided. The tremor is not caused by passive vibration from the tapping for the rate of the tremor is usually different.

The resting tremor rate is normally stable within narrow limits over a long period. Exceptions to this statement have been seen on rare occasions. Thus occasionally there was some increase in rate with voluntary stiffening or with sustained flexion and extension. The forearm flexors and extensors are in general reciprocally active, a finding that was checked on numerous occasions by running the recording paper at high speed. In one patient it was noted that with the hand in its natural position both flexors and extensors were, as is usual, reciprocally active, while when the hand was actively held in extension the rhythmic activity was confined to the extensors and when the hand was actively held in flexion the rhythmic action was confined to the flexors. Four clectromyographic channels were available for recording. When records were taken from different parts of the body showing tremor at the same time, small but definite differences of frequency were commonly encountered.

Tremor may be suppressed when the forearm muscles are tightened voluntarily. With other subjects the same manoeuvre can bring out tremor (Fig. 1). Some subjects can suppress their tremor by mental effort without showing stiffening.

BEATS PRODUCED WITH TREMOR OF THE HAND Fifty-five patients were tested for movements in the horizontal plane. All were cases of idiopathic Parkinsonism except for one in whom the disease followed carbon monoxide exposure, and one after encephalitis. The rate of the tremor at the wrist for horizontal movements on the right (41 investigations) has been $5.25 \mathrm{~Hz}$ (standard deviation, 0.78, SEM 0.12). On the left the mean rate (12 investigations) was 4.48 (standard deviation 0.54 , SEM 0.16). The difference was unexpected, and is significant.

Beats were set up most easily when the tremor was of large and stable amplitude. Satisfactory beating was seen in 50 of the 55 patients tested. In the remainder some of the difficulties stemmed from irregularities in the amplitude of the tremor. The predicted beat frequency is that of the difference in torque and tremor rates (Fig. 2). This value can be compared with the rate actually observed. An example of these data is given in the Table. The agreement is reasonably close. The increasing and decreasing parts of the beat envelope were usually symmetrical (Fig. 3). Evidently there was no tendency for the two waveforms to lock together at a particular phasing (Walsh, 1976). In three patients where the phase between the tremor and applied force was registered a regular fluctuation during each beat cycle could be seen.

The electromyographic discharges in the forearm flexors and extensors carried on during the beats. As a rule the discharges seen from the muscles during the production of beats closely matched the immediately preceding and succeeding activity when without added force the tremor 

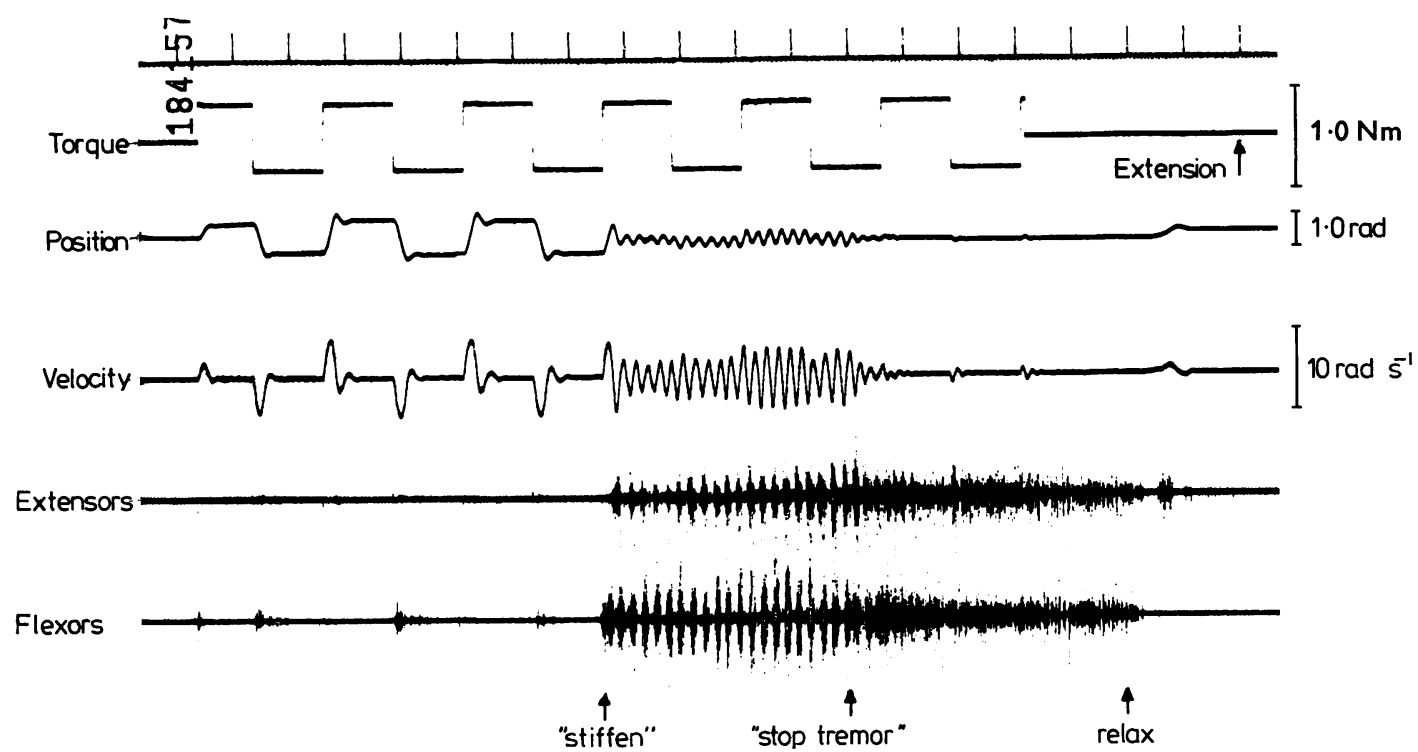

Fig. 1 Right wrist horizontal movements. Torque from motor is alternating abruptly between extensor and flexor direction at low frequency. When the patient (a 59 year old man) stiffened his wrist, the tremor of his Parkinsonism started, and the movements in response to the applied force were greatly reduced. When told to stop the tremor he could do so, and the rhythmic electromyographic discharges were replaced with continuous co-contraction. When told to relax the co-contraction was abolished.

alone was occurring. However, on occasions the discharges in both flexors and extensors were seen to become larger when the beat envelope was at its maximum. On a number of occasions, even with no spontaneous tremor, beats at once developed when the force was applied. When the force was switched off the tremor could usually be seen persisting for a while.

Eight patients were tested with the apparatus set for vertical movements. The mean frequency was $5.4 \mathrm{~Hz}$. One of the patients, aged 54 years at the time of testing, had suffered from encephalitis at the age of 8 years and the Parkinsonism dated from that time. The other cases were idiopathic. At times tremor was only seen clearly when a steady downwards force was applied. The apparatus enabled this bias to be available with or without a rhythmic sinusoid in addition. Beats to an applied rhythmic force were clearly seen in seven of the eight investigations (Fig. 4).

Two patients were tested for pronation/supination movement using the screwdriver attachment to the motor. Beats were seen on both occasions.

\section{MOVEMENTS OF THE FOOT}

Six patients were tested for tremor movements at the ankle; five had idiopathic Parkinsonism, the sixth was iatrogenic. He was psychotic and was being treated with trifluoperazine. The mean frequency was $5.4 \mathrm{~Hz}$. Beats were established to a rhythmic force in all six observations (Fig. 5).

FINGERS, ELBOW, JAWS, NECK

Three patients were investigated for tremor of a finger. In one with a tremor frequency of $5 \mathrm{~Hz}$, beats were produced without difficulty. In another patient the proximal phalanx of the index finger only existed, the rest having been blown off in the first world war. The tremor rate was $4.5 \mathrm{~Hz}$ and beats were set up. In the third patient provocative beats were set up.

One patient was tested for elbow tremor, the rate was $5 \mathrm{~Hz}$. The same rate was present in the one case of jaw tremor investigated. The rate of the head tremor for movements in the horizontal plane was $4.5 \mathrm{~Hz}$ in the one case investigated. In all three of these patients satisfactory beats were established to a rhythmic force.

\section{INERTIA}

For horizontal movements of the hand the inertia of the system could be increased by fixing a cylindrical bar in a hole in the boss connecting the motor with the potentiometer. By using bars of different length, differing amounts of inertia could be added. The tremor rate with and without 


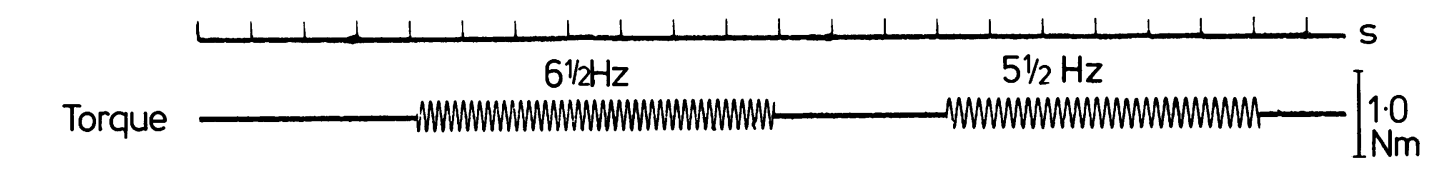

\section{$4 y_{2} \mathrm{~Hz}$

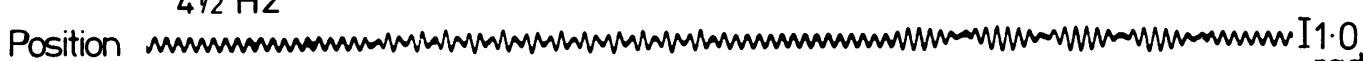

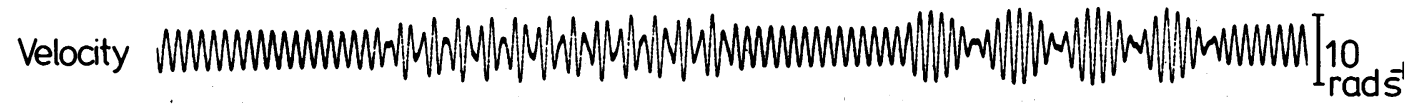

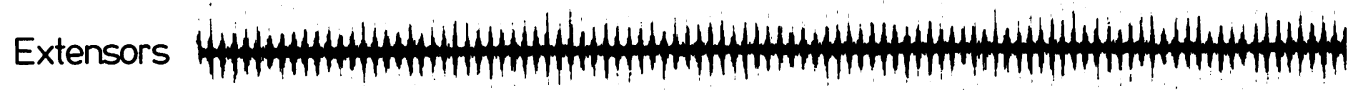

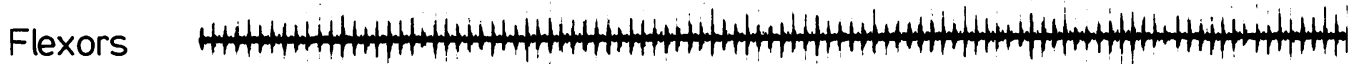

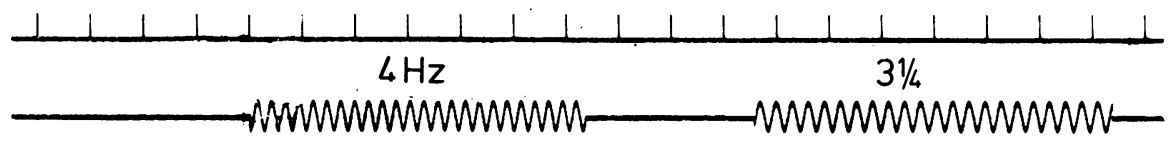

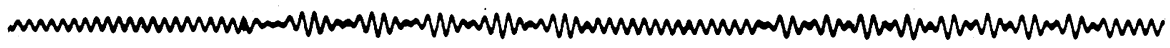

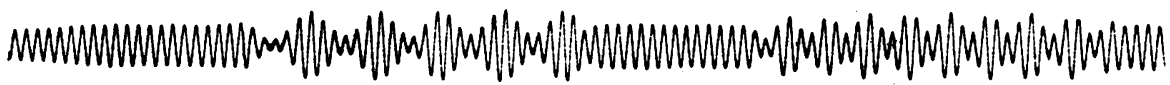

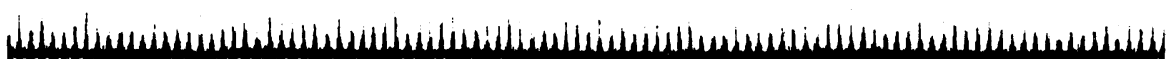

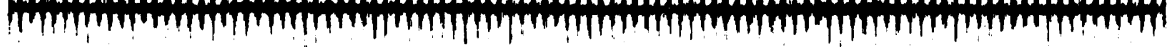

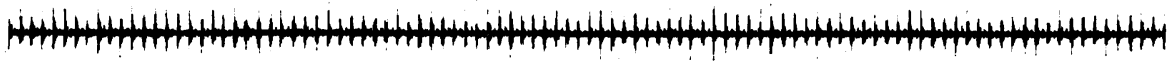

Fig. 2 Right wrist horizontal movements in a 67 year old woman. Steady Parkinsonism tremor when no torque applied, beats when torque applied. Beat envelope frequency depends on difference between frequencies of applied torque and tremor rate. Electromyogram not modulated during the beating.

Table Frequency of beats (spontaneous tremor rate $4 \mathrm{~Hz}$ )

\begin{tabular}{lll}
\hline Applied torque $(\mathrm{Hz})$ & Predicted beat $(\mathrm{Hz})$ & Observed beat $(\mathrm{Hz})$ \\
\hline 2.5 & 1.5 & 1.2 \\
3.25 & 0.75 & 0.77 \\
4.1 & 0.1 & 0.2 \\
4.9 & 0.9 & 0.6 \\
5.0 & 1.0 & 1.0 \\
5.1 & 1.1 & 1.2 \\
\hline
\end{tabular}

inertia (up to $0.02 \mathrm{~kg} . \mathrm{m}^{2}$ ) was investigated in four patients. The inertia reduced the amplitude of the tremor. In three patients there was no change of frequency. The results for the fourth were equivocal, because exceptionally the tremor rate was variable depending on the degree of voluntary stiffening. If the tremor had been caused by self re-excitation of reflexes the effect of adding inertia should have been to slow the tremor.

\section{Discussion}

Pollock and Davis (1930) described the results of posterior rhizotomy in a case of post-encephalitic Parkinsonism. They were principally concerned with the effects on rigidity. While tremor was said to remain after the operation, there are ambiguities in the account, and the significance of the findings has been a source of controversy. An attempt has been made to fill the gap by animal 


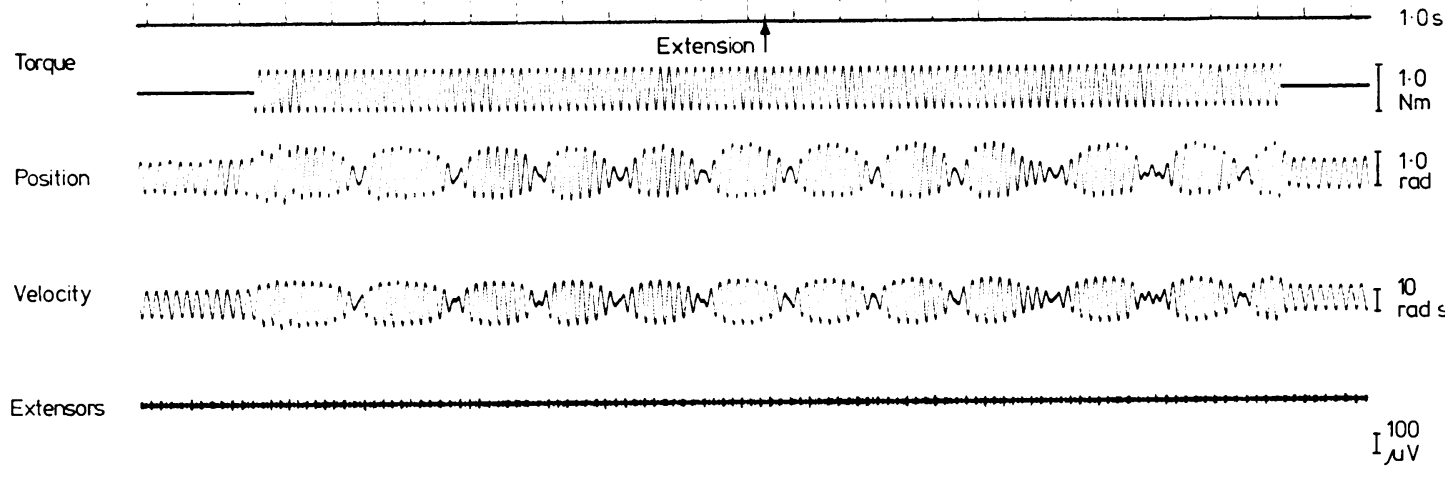

Flexors

Fig. 3 Right wrist. Slow stable beats produced between a stable tremor and a rhythmic force in a woman aged 62 years. Electromyogram is not modulated during the beating.

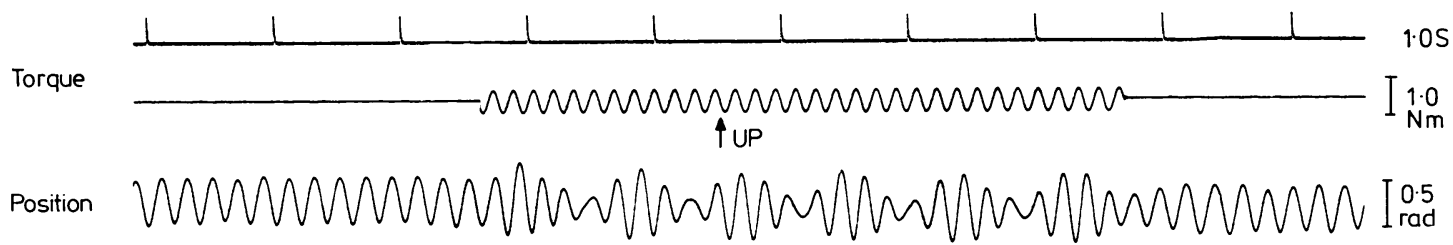

Flexors

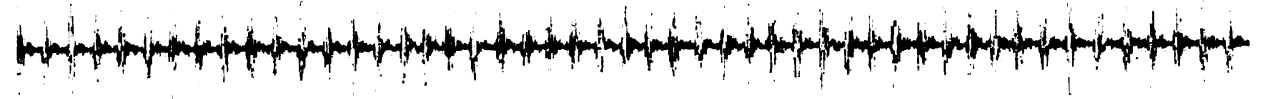

Extensors

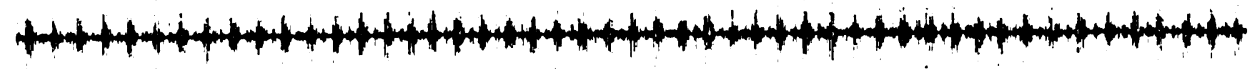

Fig. 4 Right wrist. Vertical movements in a woman aged 60 years. Beats, electromyogram is not modulated.

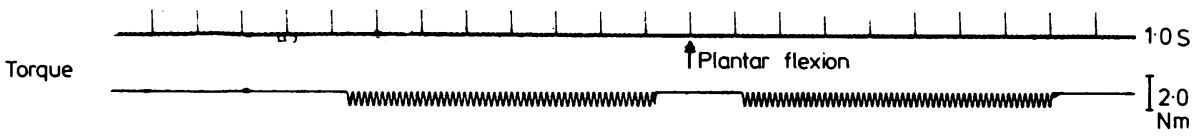

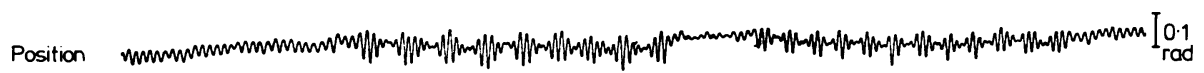

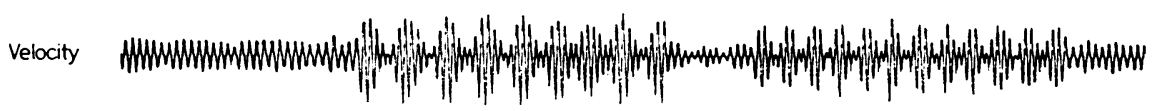

Fig. 5 Foot, horizontal movements. Beats to two rates of applied torque. Psychotic male patient, treated with trifluoperazine. 
experiments. The work has been reviewed by Lamarre (1975). Ohye et al. (1970) produced tremor in monkeys using a combination of a midbrain lesion and harmaline. Dorsal root rhizotomy did not interfere with the tremor. The reciprocity of the tremulous bursts in opposite groups of muscles was not modified by rhizotomy. These findings clearly point to a central generator as the cause of the tremor. It might be argued, however, that the tremor produced in the animal experiments is not dependent on the same mechanisms as the tremor of human Parkinsonism. Doubt may thus be cast on the relevance of the observations as well as on the interpretation of the single case report of Pollock and Davis (1930).

The results of the present study make it clear that the production of beats between an applied force and the tremor of Parkinsonism is generally possible and the rhythm is not entrained. The situation is thus comparable to an established clonus (Walsh, 1976). The results are thus strongly in favour of the view that the tremor of Parkinsonism results from the activity of a central generator or generators. Records of muscle spindle activity in patients with the tremor of Parkinsonism have been obtained by Hagbarth et al. (1975). Two bursts of activity may be seen for each beat of the tremor, and it has been concluded that the indirect loop through the spindles takes no part in the initiation of a beat, for the electromyographic bursts start before the initiation of the spindle contraction discharges. Hagbarth et al. considered it possible that the spindle afferent discharge during contraction might influence later parts of the tremor burst. The present data bear on this point for the electromyographic discharge was never increased when the force of the motor was opposing the tremor and producing a minimum in the beat envelope. It appears clear that the tremor is neither servo driven nor servo assisted.

There is evidence that the tremor of Parkinsonism may be reset by peripheral nerve stimulation and this might be considered as being evidence against the conclusions of the present work. It has been pointed out that "nerve stimulation, however, affects all levels of the nervous system ..." (Mones and Weiss, 1969). The results are applicable only to resting tremor. Action tremor may also be present in Parkinsonism and has not been investigated systematically in this study. It may well behave differently.
Royal Society (government) grants and funds from Roche Products Ltd, are gratefully acknowledged. The apparatus was constructed by Mr G. Wright.

\section{References}

Dinakar, I., and Walsh, E. G. (1973). Parkinsonismtwo types of activation. Journal of Physiology, 231, 31-32P.

Gillingham, J., Walsh, E. G., and Tsukamoto, Y. (1972). Treatment of rigidity. In Parkinson's Disease, vol. 1, pp. 93-114. Edited by J. Siegried. Huber: Bern.

Hagbarth, K. E., Wallin, G., Lofstedt, L., and Aquilonius, S. M. (1975). Muscle spindle activity in alternating tremor of Parkinsonism and in clonus. Journal of Neurology, Neurosurgery, and Psychiatry, 38, 636-641.

Hufschmidt, H. J. (1963). Proprioceptive origin of Parkinsonism tremor. Nature, 200, 367-368.

Hufschmidt, H. J. (1959). Die reflektorische Grundlage des Parkinsontremors. Deutsche Zeitschrift für Nervenheilkunde, 179, 298-308.

Lamarre, Y. (1975). Tremorgenic mechanisms in primates. In Advances in Neurology, vol. 10, pp. 23-34. Edited by B. S. Meldrum and C. D. Marsden. Raven Press: New York.

Mones, R. J., and Weiss, A. H. (1969). The responce of the tremor of patients with Parkinsonism to peripheral nerve stimulation. Journal of Neurology, Neurosurgery, and Psychiatry, 32, 521-518.

Ohye, C., Bouchard, R., Larochelle, L., Bédard, P., Boucher, R. Raphy, B., and Poirier, L. J. (1970). Effect of dorsal rhizotomy on postural tremor in the monkey. Experimental Brain Research, 10, 140-150. Pollock, L. J., and Davis, L. (1930). Muscle tone in Parkinsonism states. Archives of Neurology and Psychiatry (Chicago), 23, 303-319.

Stiles, R. N., and Pozos, R. S. (1976). A mechanical reflex oscillator hypothesis for Parkinsonism hand tremor. Journal of A pplied Physiology, 40, 990-998.

Walsh, E. G. (1969). Interference with the tremor of Parkinsonism by the application of a rhythmic force. Journal of Physiology, 202, 109-110P.

Walsh, E. G. (1974). Pugnatron-like postural reactions in a patient with familial dystonia-torque induced motion analysis. Journal of Neurology, Neurosurgery, and Psychiatry, 37, 559-565.

Walsh, E. G. (1976). Clonus: beats provoked by the application of a rhythmic force. Journal of Neurology, Neurosurgery, and Psychiatry, 39, 265274.

Walsh, E. G. (1978). Parkinsonism tremor-production of beats by the application of a rhythmic force. Journal of Physiology, 278, 40-41P. 